\title{
An Overview of Robotics in Functional Neurosurgery
}

\author{
Vikas Vazhayil $^{1}$ Madhav Rao² Manish Beniwal' Nishanth Sadashiva ${ }^{1}$ \\ Narasinga Rao Kannepalli Venkata Lakshmi ${ }^{1}$ Sampath Somanna ${ }^{1}$
}

\author{
${ }^{1}$ Department of Neurosurgery, National Institute of Mental Health \\ and Neurosciences (NIMHANS), Bengaluru, Karnataka, India \\ International Institute of Information Technology, Bangalore, \\ Karnataka, India
}

Indian J Neurosurg 2019;8:6-10

\author{
Address for correspondence Vikas Vazhayil, MBBS, MS General \\ Surgery, MCh Neurosurgery, DNB Neurosurgery, (PhD Surgical Robotics), \\ Additional Professor of Neurosurgery, Quarter No. 44, Type 5, Block 3, \\ BRC Campus, NIMHANS Residential Quarters, Dharmaram P.O., \\ Bengaluru 560029, Karnataka, India (e-mail: vikas.drv@gmail.com).
}

\author{
Abstract \\ Keywords \\ - functional \\ neurosurgery \\ - robotic neurosurgery \\ - surgical error \\ - stereotactic \\ procedures
}

Stereotactic techniques are used in a wide range of neurosurgical procedures. The procedures demand a high degree of spatial accuracy and minimal error. There are diverse functional surgeries that require stereotactic procedures, including deep brain stimulation, brain biopsies, and epilepsy procedures. Though the disease processes are diverse, all these procedures require accurate targeting of deep structures without visual guidance. The use of robots for stereotactic procedures is a natural progression in the surgeon's quest for higher accuracy and lower complications. This paper reviews the role of robots in stereotactic procedures and outlines current status of robots in stereotactic procedures. The shortcomings of current systems and an outline of an ideal stereotactic device are presented.

\section{Introduction}

Robotic surgery has made inroads into multiple specialties. Over the past decade, several common laparoscopic and thoracic procedures can be performed using master-slave robotic devices. Neurosurgery has been a late adopter of robotics. General-purpose surgical robots have not found neurosurgical applications. The specialized anatomical constraints and risks of neurosurgery have been responsible for the delay in research in this field. Of the various neurosurgical procedures, functional procedures have caught maximum attention in this field. This is due to the nature and exacting requirements of these procedures.

\section{An Overview of Robotics}

The growth of industrial robotics happened in the late 1960s with the establishment of dedicated companies that made multi-jointed master-slave systems. The human masters control the "slave" robotic device and execute the procedure. By this concept, human surgeons control all aspects of the procedure. The robot facilitates accuracy, task repeatability with minimal error, and consistency. The multi-jointed robot has been the traditional model on which most modern surgical robots are designed. A robot generally can be classified according to several features. They can be classified into serial and parallel robots based on the organization of elements within the robot. Parallel robots have greater structural received

September 25, 2018

accepted

October 29, 2018

published online

April 22, 2019 stability and are more accurate than serial robots, which is of relevance for neurosurgical stereotactic procedures. They can also be classified based on their number of degrees of freedom or axes. The degrees of freedom basically indicates the capability of a robot. ${ }^{1}$ The joints between these elements can be classified into prismatic (sliding or linear joint), revolute joints (hinge joint) and screw joints (helical joint). Other types of joints include cylindrical, spherical planar pair, and Hooke joint. The important joints are highlighted in the diagrams ( - Fig. 1).

Prismatic joints allow for one directional motion and are relatively error free. All these joints have one degree of freedom. Examples of the joints with multiple degrees of freedom include cylindrical, universal, and spherical (ball and socket) joints. Based on the joints and the nature of kinematics, robotic devices are said to have varying degrees of freedom ( - Fig. 2).

\section{Surgical Robotics}

Different surgical robotic systems have differing arms based on the target use. Thus, robots such as Da Vinci that are used for laparoscopic surgery have actuator arms designed for laparoscopic use with small gripping end tools based on an "EndoWrist." Robots used for orthopaedic procedures, thus having tools built to position and drill into long bones. In essence, surgical robots have differing designs based on the surgical requirements. At present there is no single
License terms

Society of India
DOI https://doi.org/

10.1055/s-0039-1687715 ISSN 2277-954X. 


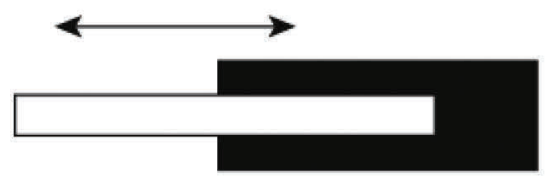

Prismatic Joint

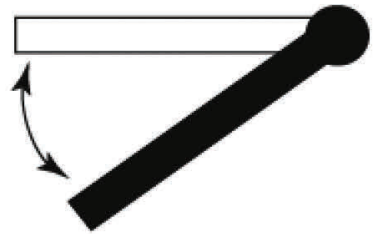

Hinge Joint

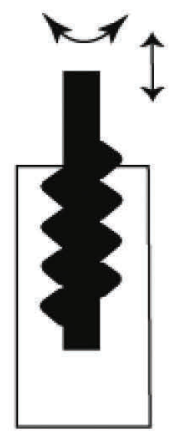

Helical Joint

Fig. 1 Example of joints used in robots and the movements associated with each joint.

general-purpose surgical robot that can be used across all surgical specialties. Though these devices are known as surgical robots, and the procedure-labeled robotic surgery, robots are not used in the complete surgical procedure. A significant component of the procedure is still performed by humans. For example, in laparoscopic surgery, human surgeons have to perform peritoneal insufflation and primary viewing only after which the robot docks with the abdominal ports. Similarly, the orthopaedic robots are used only after bone exposure is completed. A surgeon requires specialized training to operate a surgical robot. The surgeon is thus the master of the surgery and the robot a slave that executes the tasks. The current surgical robots are no more than action filtering devices with minimal autonomous function. Thus, they behave as advanced tools that simplify a complex task, for example steps such as laparoscopic suturing that otherwise have a protracted learning curve. Thus, the primary role of master-slave robots is in reducing the skill level required to execute a particular task.

\section{Neurosurgery and Robotics}

The primary purpose of using robots in neurosurgery has been to improve targeting of deep structures. Targeting of deep structures, especially for biopsy, has been a focus of early robotic devices. The first of these procedures were incidentally performed using an industrial robot. ${ }^{2,3}$ Robotic targeting has high precision, and this was the primary motivation for utilizing them. Surface surgery or surgeries requiring a formal craniotomy requires general purpose robots. These robots require arms, master-slave control systems, and sophisticated safety and feedback mechanisms. The first dedicated attempt to build a general-purpose neurosurgical robot was by Prof. Sutherland and his team at the University of Calgary. ${ }^{4,5}$ Several other groups notably in Japan and Europe have been working on general-purpose neurosurgical robots. However, stereotactic robots have more commercial success than general-purpose neurosurgical robots.

\section{Functional Neurosurgery}

A wide range of neurosurgical procedures come under the gamut of functional neurosurgery. They include procedures for movement disorders such as deep brain stimulation (DBS), epilepsy surgery including resective and ablative procedure, and procedures for pain and psychiatric diseases. Functional neurosurgery is characterized by the following unique features. The targets are functional neuronal tissue. The targets may be on the surface or deep within the brain
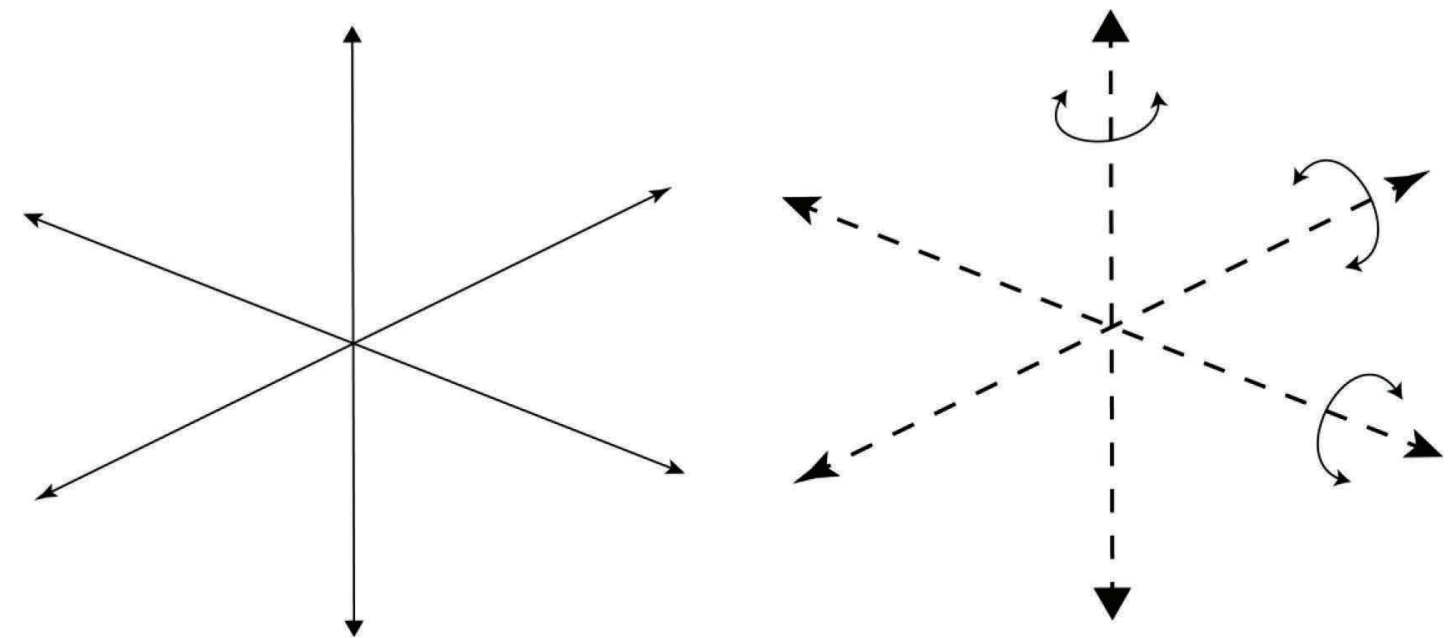

Fig. 2 Depiction of degrees of freedom associated with an object. The primary directions are listed on the left-hand diagram. The rotary movements are depicted in the right-hand diagram constituting the six degrees of freedom for a given object. 
substance. Functional procedures are also performed on spinal cord. Surgical technique requires targeting of these tissues; especially in the brain, it requires millimetric precision. Procedures for DBS and pain require placement of biocompatible hardware in precise locations. The important common factors in such procedures include localization by high-resolution magnetic resonance imaging (MRI) and high accuracy in reaching anatomical targets. Stereotactic localization has been the gold standard in localizing small targets within the substance of the brain. The standard procedure involves imaging with a rigid calibrated metallic frame fixed to the skull. The frame-based coordinate system ensures that errors are minimized and the target is reached with high accuracy. Frame-based localization revolutionized target localization within the brain. Thus, targets could be acquired and localized without visual confirmation and with a high accuracy. The targets are calculated in relation to the coordinate system of the frame. Path planning features are available in most modern image-viewing software. High-resolution 3-Tesla MRI has become standard of care for obtaining imaging for functional procedures. Improvements in imaging have been responsible for improved localization of targets such as the subthalamic nucleus. Image fusion techniques by which MRI and CT (computed tomography) images can be fused have made significant improvements in reducing procedure time. Electrophysiologic recording has also improved target localization by adding a physiologic component. Adverse effects by damage to normal structures have been minimized by electrophysiologic monitoring during the procedure. In essence, a gradual improvement in several procedural aspects of functional neurosurgery has been evident.

\section{Target Localization and Reachability}

An important component of functional neurosurgery is in reaching targets deep within the brain. These targets are visualized by high-resolution MRI. Conventionally, the stereotactic frame-based calculation is used to reach a target within the brain. The frame provides a volume within which a given target can be localized. Rigid fixation of the skull to the frame ensures that the volume within the skull that is not visible can be reached by using mathematical calculation. The co-registration of the frame and skull volume is done by obtaining high-resolution MRI. Newer techniques such as image fusion help reduce overall procedure time. The basic premise remains that the volume within the skull is imaged in relation to the "fixed" frame. The calculated targets are reached through a set of precision instrumentation mounted on the same stereotactic frame. The frame is thus a bridge between the intracranial target and operating tools.

The intracranial targets are small in size and are surrounded by eloquent structures, damage to which results in significant neurologic deficits. The targeting robot thus requires the following features. It has to have a small profile to prevent damage to normal structures surrounding the target. There should be an opening in the skull such that the device can safely traverse without any change in the path to the target. The varying consistency of the brain and brain-shift are factors that can cause change in trajectory. A major issue of concern is that the target is not directly visualized at any point of the surgery. Thus, the error in reaching the target can only be computed. Indirect means such as microelectrode recordings are indirect proxies for reducing error by means of electrical confirmation of the target region. The occurrence of error in targeting, its identification, and correction form an important application of robotics in functional neurosurgery. An understanding of what constitutes error is thus needed.

\section{Error and Its Minimization in Functional Neurosurgery}

A complete DBS surgical procedure involves skin incision, dissection, burr-hole placement, dural incision, electrode array placement and recording, and permanent electrode placement followed by wound closure. The procedures of frame fixation, image acquisition, fusion, and registration occur prior to the actual surgery. The time from burr-hole placement to burr-hole closure after the electrode fixation is crucial and has the maximum risk of workflow error. The final trajectory from the skin surface to the target tissue is linear. The end actuator system for this phase of surgery has to be strictly linear with one direction of freedom. Prismatic or helical joints are ideal joints at the last set of effectors in contact with the skull. The number of joints in relation to the skull has to be minimized to reduce errors. The size of the subthalamic nucleus is approximately $3 \mathrm{~mm}$. Even if a $10 \%$ error margin is considered, the device should be able to reach a given spatial target with an error less than $0.3 \mathrm{~mm}$ from the target tissue in 3D space. Current documentations of error during stereotactic procedures are in the range of $2 \mathrm{~mm} .{ }^{6,7}$ Newer techniques and developments are expected to reduce the errors further as more and more automation enters into the workflow. ${ }^{5,8,9}$ Sources of error in robotic stereotactic procedures have been studied in detail. ${ }^{6,10}$ These include imaging resolution, distortions in image acquisition and the display system, anatomical changes that may occur between image acquisition and the actual surgery; registration errors between patient and the imaging; camera, tool, and robot calibration errors; and kinematic errors. Regarding stereotactic brain procedures, the access path from the dural entry point to the target is blind. There is no visual confirmation regarding the accuracy of having reached the target. This is somewhat mitigated by image guidance systems and microelectrode recordings for DBS procedures. These techniques in turn have been advancements on the earlier techniques of brain atlas-based localizations. Even with current (nonrobotic) practices, the issues of brain-shift, frame errors, and image acquisition issues persist to varying extents. It is in this context that robots would play a major role in the near future in minimizing error.

Developing a single robot that can perform skin incision, scalp dissection, and retraction is a difficult proposition. Benefits of developing tools to this end are not of relevance, as the chances of error occurring during these steps are minimal. From the point of burr-hole placement to the electrode implant stage, robotic devices can minimize error. It is thus imperative that a fully dedicated functional neurosurgery robot should have multiple components dedicated to diverse 
tasks such as burr-hole placement, dural incision, prevention of intradural air entry, and electrode array deployment and implantation. Some of the tasks may require dedicated separate modules, for example drills, and haemostatic devices. The other elements such as the electrode array, electrode implantation, and testing components are best designed coaxial and brought into play at relevant points of time. The surgical steps after burr-hole placement up to electrode delivery and implant delivery have to be a seamless robotic procedure.

\section{Robots in Functional Neurosurgery}

Of various surgical procedures undertaken in neurosurgery, highest benefits of robotic intervention can happen in functional neurosurgery. Though a plethora of robotic surgical programs exist, ${ }^{8,11-14}$ commercially available robots are few and far between. A relatively large number of stereotactic robotic programs have been documented in the literature. However, conversion of research programs to commercially successful products has been noted to be very few. The Neuromate Stereotactic robot (Integrated Surgical Systems), Renaissance system (Mazor Robotics), and ROSA (Medtech) are commercially available and designed for neurosurgical stereotactic procedures. Neuromate was the first to obtain U.S. Food and Drug Administration (FDA) approval for stereotactic neurosurgical procedures. All these systems have been used for functional neurosurgical procedures. The Ronna G3 (University of Zagreb, Croatia) ${ }^{7}$ and iSYS1 (iSYS Medizintechnik) $)^{5}$ are newer systems for stereotactic applications.

\section{Indian Groups}

Surgical robotic research has not achieved institutional status in India. Individual projects on various aspects of surgical robotics have been conducted with a focus on assist devices and simulators. Bhabha Atomic Research Centre in collaboration with Tata Memorial Hospital, Mumbai, have developed a stereotactic robot for neurosurgical applications. ${ }^{2}$ The device has six degrees of freedom and has a parallel kinematic mechanism and is undergoing clinical testing. The Surgical and Assistive Robotics Laboratory (SARL) is a joint effort of NIMHANS (National Institute of Mental Health and Neurosciences) and IIIT (International Institute of Information Technology) Bangalore. Though the focus of the group is on hyperflexible surgical robots, visualization, and ranging, linear targeting systems are also in the process of development. Regarding the linear targeting device, the development philosophy envisages both cranial and spinal applications encompassing stereotactic and spinal positioning systems. The work on the linear targeting system is at an early stage, and a few prototype devices have been developed: image integration and mapping. More and more hospitals in both government and private sectors have been using commercially available stereotactic robots for neurosurgical procedures. At the time of this writing, two Indian centers have been listed on the ROSA Web site. Mazor robots have also been reportedly used in two private sector hospitals for spine applications.

\section{Discussion}

Attempts at using robotic technology for neurosurgical procedures have started nearly three decades back. However, the number of research programs translating into commercial products has been few. The cost of a dedicated robotic surgery program is enormous and is in the range of several million dollars. The cost of the device development and marketing thus forms a big barrier for most research groups. Unlike in laparoscopic surgery where the "EndoWrist" of the Da Vinci system had a distinct surgical utility, current stereotactic robotic neurosurgical devices do not provide a distinct advantage over a meticulous human surgeon. Studies on outcome differences between robotic and human neurosurgery have not been conducted. It may be a little too early to conduct these studies as the technologies, and the current robotic surgical workflows have not yet matured. The robots developed are linear devices with applications restricted to image guidance and stereotactic procedures. Thus, two factors determine the popularity of robotic surgical procedures: cost and significant value addition. Value addition can be in terms of ease of use, decrease in time of procedure, or an actual reduction in cost. An ideal functional robot should seamlessly perform a complete functional neurosurgery procedure with minimal human intervention. ${ }^{13}$ One of the major concerns of contemporary DBS procedures is the necessity of performing surgery awake. The procedure also is known to last several hours. At a conceptual level, the entire set of surgical tasks involves accessing the target region with multiple passes of electrodes and recording. It is this part of surgery that a robot with its inbuilt accuracy and check systems would be able to reduce significantly and perform consistently with higher accuracy. Simultaneous (bilateral) performance of the procedure can be achieved with robotic assistance further reducing overall procedure time. Only when ease of use parameters compared with current nonrobotic procedures along with a decrease in pricing occur would an inflexion point occur for robotic neurosurgery. In terms of robotics, technical challenges for functional neurosurgery are minimal, and in that, no new line of research is required for developing an ideal robot. However, from the point of functional neurosurgery, significant advances have to occur over and above the current workflows offered by the robotic systems. The challenges lie in design and not as much in device development or research. As it stands today, the elements required for an ideal stereotactic robotic system are already in place. The coming together of these elements has not occurred till date. The key goal to be achieved would be in minimizing human intervention during the procedure. In an ideal system, human presence would only be supervisory following the planning stage. Only when human intervention is minimized during the procedure will the full benefits of robotic intervention would become apparent. This is not only in the context of accuracy but also in minimizing cost per procedure and in ease of conducting the procedure. These factors in turn would reduce the overall costs. Ultimately, economic considerations play a major role in adaptation of the novel procedure. 
An ideal surgical master-slave robot for functional neurosurgery should have the following features. The robot should be able to execute all position and spatial targeting related steps with minimal human intervention. The robot must be able to perceive and correct for errors at all phases of the operative procedure. The user interface should be simple enough that no specialized training would be required to operate the robot. A favorable robotic surgical workflow would require less technical demand on the individual surgeon. Especially in functional surgical procedures, a well-designed robot would reduce the skill level required of the surgeon for performing the procedure. More surgeons can thus undertake the procedure using the robot ultimately benefitting patients who otherwise have to travel to high-volume centers for a good outcome.

\section{Conclusion}

The scope of robotics in functional neurosurgery is immense. As implants for neurologic diseases evolve, the demand for target-based neurosurgical interventions will increase. Robots would ensure that high-precision surgery can be performed even in low-volume centers. Robotic surgery would be the leveler, ensuring widespread availability of surgical options. As with any emerging technology, cost is a major issue of concern. However, as more and more research and commercial groups enter into the field and the market opens out, the only direction the cost can move is down. Ideal robotic devices would supplement and complement the surgeon's knowledge and reduce the skill and effort levels required for high-level consistent performance. That ultimately is the advantage of robotic surgery. Robots are advanced tools. This tool in the hands of trained surgeons would help in complementing human efforts for the surgical management of functional neurosurgical disorders.

\section{Acknowledgments}

None.

Conflict of Interest

None.

\section{References}

1 Ashitava G. Robotics: Fundamental Concepts and Analysis. New Delhi, India: Oxford University Press; 2006
2 Kaushik A, Bhutani G, Venkata PPK, Dwarakanath TA, Moiyadi A. Image based data preparation for neuronavigation. 2nd Int 17th Natl Conf Mach Mech Ina 2015. 2015:1-9. https://www.scopus.com/inward/record.uri?eid=2-s2.0-8501 5174336\&partnerID=40\&md5=6e41228898bb547ad4f9ade$564 \mathrm{~b} 2 \mathrm{e} 380$

3 Kwoh YS, Hou J, Jonckheere EA, Hayati S. A robot with improved absolute positioning accuracy for CT guided stereotactic brain surgery. IEEE Trans Biomed Eng 1988;35(2):153-160

4 Sutherland GR, McBeth PB, Louw DF. NeuroArm: an MR compatible robot for microsurgery. Int Congr Ser 2003;1256:504-508

5 Sutherland GR, Wolfsberger S, Lama S, Zarei-nia K. The Evolution of neuroArm. Neurosurgery. 2013;72(suppl 1):A27A32. http://dx.doi.org/10.1227/NEU.0b013e318270da19

6 Widmann G, Schullian P, Ortler M, Bale R. Frameless stereotactic targeting devices: technical features, targeting errors and clinical results. Int J Med Robot 2012;8(1):1-16

7 Dlaka D, Švaco M, Chudy D, et al. Brain biopsy performed with the RONNA G3 system: a case study on using a novel robotic navigation device for stereotactic neurosurgery. Int J Med Robot 2018;14(1):1-7

$8 \mathrm{Xu} F$, Jin $\mathrm{H}$, Yang X, et al. Improved accuracy using a modified registration method of ROSA in deep brain stimulation surgery. Neurosurg Focus 2018;45(2):E18

9 Suligoj F, Svaco M, Jerbic B, Sekoranja B, Vidakovic J. Automated marker localization in the planning phase of robotic neurosurgery. IEEE Access 2017;5:12265-12274

10 Finlay PA, Morgan P. Pathfinder image guided robot for neurosurgery. Ind Rob 2003;30(1):30-34

11 Marcus HJ, Vakharia VN, Ourselin S, Duncan J, Tisdall M, Aquilina K. Robot-assisted stereotactic brain biopsy: systematic review and bibliometric analysis. Childs Nerv Syst 2018; $\cdots: 1-11$

12 Correa-Arana K, Vivas-Albán OA, Sabater-Navarro JM, Correa-Arana K, Vivas-Albán OA, Sabater-Navarro JM. Neurosurgery and brain shift: review of the state of the art and main contributions of robotics Neurocirugía y desplazamientos cerebrales: una revisión del estado del arte y principales contribuciones desde la robótica Cómo citar/How to cite. 2017;20(40):123-7799. http://www.scielo.org.co/pdf/teclo/ v20n40/v20n40a10.pdf

13 Faria C, Erlhagen W, Rito M, De Momi E, Ferrigno G, Bicho E. Review of robotic technology for stereotactic neurosurgery. IEEE Rev Biomed Eng 2015;8:125-137

14 Dogangil G, Davies BL, Rodriguez y Baena F. A review of medical robotics for minimally invasive soft tissue surgery. Proc Inst Mech Eng H 2010;224(5):653-679 